\title{
Coronavirus Disease-19 Pandemic and Undergraduate Medical Students Teaching/Learning
}

\section{Vakhtang Shoshiashvili*}

Anesthesiologist, First University Clinic, TSMU and Assistant Professor, Faculty of General Medicine, TSMU and Associate Professor, Faculty of Medicine, European

University, Associate Professor, Faculty of Medicine, Kutaisi University, Georgia

*Corresponding Author: Vakhtang Shoshiashvili, Anesthesiologist, First

University Clinic, TSMU and Assistant Professor, Faculty of General Medicine, TSMU and Associate Professor, Faculty of Medicine, European University, Associate Professor, Faculty of Medicine, Kutaisi University, Georgia.

Since the beginning of 2020 coronavirus disease-19 (COVID-19) pandemic caused by a novel coronavirus all aspects of life globally have been affected, including teaching/learning.

According to epidemiologic situations in most countries medical universities/colleges/schools had begun teaching/learning online. Adaptation to this situation was not easy, but already existing practice of video lectures, conferences, telemedicine, webinars, different electronic platforms come for help. Previously all of this served as an adjunct to the traditional learning process and suddenly situation was changed dramatically. Due to spread of pandemic and epidemiologic restrictions, online learning became a routine practice. Is it equal to the traditional teaching/learning process and how will this situation influence the professionalism and career of future doctors? It is difficult to answer this question. At first, preclinical education had been already practiced online in developed countries. For example, anatomy education in some universities of UK is providing with online resources using YouTube channel, Instagram and website since 2014 [3]. This practice now is spreading worldwide, but problems stays for clinical teaching/ learning, because the absence of real clinical practice is unavoidable during online process. On the other hand, due to feeling the luck of clinical practice, is rising students interests in residency, because potentially it gives the chance to get the sufficient clinical practice in future profession. Therefore, student motivations of learning must rise during COVID-19 pandemic.

Consequently, the teaching practice and rising responsibility of the teacher is changing too.
Received: May 26, 2021

Published: July 12, 2021

(C) All rights are reserved by Vakhtang

Shoshiashvili.
Virtual clinical simulations, virtual hospital, video lectures and presentations became routine in teaching of medicine. This online learning supports students to understand different clinical situations, make decisions, view and assess the results of diagnostic, therapeutic or interventional actions, which is possible to provide during the digital simulation. It motivates students to learn more, again on the basis of e-books, e-lectures, e-presentations. The result is that, the students can themselves prepare the presentation or discussion on an interesting theme and feel like a skilled doctor, can answer many questions, but...without the real patient and communications. This social isolation makes the student lonely and vulnerable. For improving of these psychological problems and reducing stress and anxiety, students are forming groups according to their choices $[2,4]$. Therefore they are adapting with online learning, moreover, some of them prefer it in comparison to the traditional clinical learning. Despite of this, it is well established, that for proper education student must be involved in real clinical practice and work alongside with experts [5]. For this purpose, students' clinical volunteering is very important [1].

We must conclude, that in the absence of patient contact, different electronic platforms are good alternatives for medicine teaching/learning. Student and teacher motivation and clinical volunteering is very important during online learning. In some circumstances, according to the local student/teacher preferences this new practice of online teaching/learning might be effectively usable in future, after COVID-19 pandemic too. 


\section{Bibliography}

1. Byrne MHV., et al. "COVIDReady2 study protocol: cross- sectional survey of medical student volunteering and education during the COVID-19 pandemic in the United Kingdom". BMC Medical Education 21 (2021): 211.

2. Chatterjee S. "The COVID-19 Pandemic Through the Lens of a Medical Student in India". International Journal of Medical Students 8.1 (2020): 82-83.

3. Hall S and Border S. "Online Neuroanatomy Education and Its Role During the Coronavirus Disease 2019 (COVID-19) Lockdown". World Neurosurgery 139 (2020): 628.

4. Hamad SMS., et al. "To teach is to learn twice" Added value of peer learning among medical students during COVID-19 Pandemic". MedEdPublish 9.1 (2000): 1-7.

5. Li LC., et al. "Evolution of Wenger's concept of community of practice”. Implementation Science 4.1 (2009): 11.

Volume 5 Issue 8 August 2021

(C) All rights are reserved by Vakhtang Shoshiashvili. 